\title{
KMOS @ the VLT: Commissioning and Early Science
}

\author{
Ray Sharples ${ }^{1}$ and the KMOS Team $\dagger$ \\ ${ }^{1}$ Department of Physics, University of Durham, Durham, UK \\ email: r.m.sharples@durham.ac.uk
}

\begin{abstract}
KMOS is a multi-object near-infrared integral field spectrograph built by a consortium of UK and German institutes for the ESO Paranal Observatory. KMOS completed commissioning at the ESO VLT in Spring 2013 and was made available to the user community from Oct 2013. We present the unique capabilities of KMOS for 3D galaxy surveys and report on the on-sky performance verification measured during three commissioning runs on the ESO VLT in 2012/13 and some of the early science results.
\end{abstract}

Keywords. instrumentation, infrared spectroscopy, integral field spectroscopy

\section{Introduction}

KMOS is a unique cryogenic near-infrared $(0.8$ to $2.5 \mu \mathrm{m})$ multi-object spectrograph that uses deployable integral field units (d-IFUs) to obtain spatially-resolved spectra for multiple targets selected from within an extended field of view. The top-level scientific requirements are: (i) to support spatially-resolved (3-D) spectroscopy; (ii) to allow multiplexed spectroscopic observations; (iii) to allow observations across any of the IZ, YJ, $\mathrm{H}$, and $\mathrm{K}$ infrared atmospheric windows in a single exposure. These requirements have been flowed down to the final system design specifications (Sharples et al. 2004). The final design employs 24 robotic arms that position fold mirrors at user-specified locations within a 7.2 arcmin diameter field-of-view. Each arm selects a sub-field on the sky of $2.8 \times 2.8$ arcseconds. The size of these sub-fields is chosen to match the compact sizes of high redshift galaxies, with a spatial sampling of 0.2 arcsec per pixel to properly sample the excellent infrared seeing at Paranal (median FWHM $\sim 0.5$ arcsec in the K-band). The sub-fields are optically relayed to 24 advanced image slicer d-IFUs that partition each sub-field into 14 slices with 14 spatial pixels along each slice. Light from the output of the d-IFUs is dispersed by three cryogenic grating spectrometers which generate $14 \mathrm{x} 14$ spectra with $\sim 1000$ Nyquist-sampled spectral resolution elements for all of the 24 independent sub-fields. The spectrometers each employ a single $2 \mathrm{kx} 2 \mathrm{k}$ Hawaii-2RG $\mathrm{HgCdTe}$ detector and are each fed by 8 d-IFUs. The optical layout for the whole system has a threefold symmetry about the Nasmyth optical axis which has allowed a staged/modular approach to assembly, integration and test. The following sections present the technical overview of the instrument and the as-built performance during commissioning and science verification.

$\dagger$ Ralf Bender, Alex Agudo Berbel, Richard Bennett, Naidu Bezawada, Roberto Castillo, Michele Cirasuolo, Paul Clark, George Davidson, Richard Davies, Roger Davies, Marc Dubbeldam, Alasdair Fairley, Gert Finger, Natascha Frster Schreiber, Reinhard Genzel, Reinhold Haefner, Achim Hess, Ives Jung, Ian Lewis, David Montgomery, John Murray, Bernard Muschielok, Jeff Pirard, Suzanne Ramsey, Phil Rees, Josef Richter, David Robertson, Ian Robson, Stephen Rolt, Roberto Saglia, Ivo Saviane, Joerg Schlichter, Linda Schmidtobreik, Alex Segovia, Alain Smette, Matthias Tecza, Stephen Todd, Michael Wegner, Erich Wiezorrek and others. 

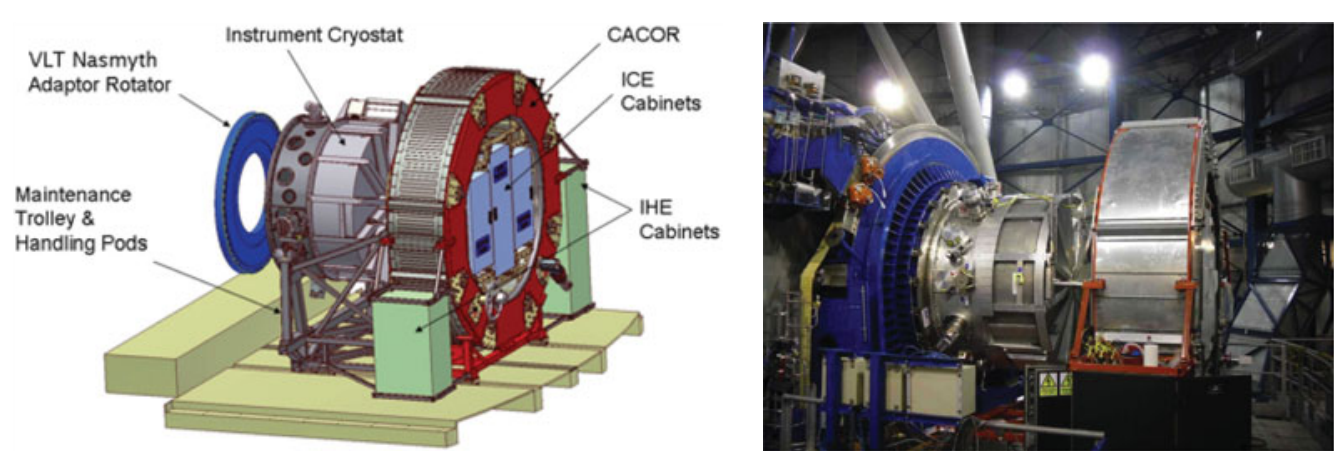

Figure 1. Schematic view of KMOS (left) and an image of KMOS on the Nasmyth platform of VLT UT1 (right).

\section{Technical Description}

\subsection{Hardware Overview}

From a hardware perspective the instrument neatly partitions into the following key subsystems:

- Pickoff subsystem

- IFU subsystem

- Spectrograph subsystem

- Detector subsystem

- Infrastructure subsystems and electronics

The opto-mechanical parts of the instrument are all contained within a single cryostat mounted on the instrument rotator at the Nasmyth focus of the VLT UT1 telescope. The total rotating mass is $2500 \mathrm{~kg}$ of which $1100 \mathrm{~kg}$ is the cold mass. At the temperature and pressure conditions on Paranal, the optical bench was stabilized at a temperature of $115 \mathrm{~K}$. Because of the mass-limit on the instrument rotator $(3000 \mathrm{~kg})$ the electronics cabinets are mounted on a separate instrument rotator (CACOR) sitting on the Nasmyth platform (see Fig. 1). The total mass during operations is $6000 \mathrm{~kg}$. KMOS was designed to have three identical modules, each of them comprising eight pickoff arm systems, eight d-IFUs, one spectrograph and one near-IR detector. This ensures that in case of failure of one system, $2 / 3$ of the functionality will still remain. The following sections briefly describe the different sub-systems of KMOS in the order they are encountered along the optical path going from the telescope to the detector; further details can be found in Sharples et al. (2006).

\subsection{Pickoff System}

The selection of targets is achieved by means of 24 cryogenic $r-\theta$ robots (pickoff arms), which together patrol a 7.2 arcmin field of view. This is located inside the cryostat below a vacuum-spaced doublet corrector which produces a flat telecentric focal plane. The pickoff arms are arranged into two different layers (top and bottom) of twelve arms each patrolling on either side of the focal plane, so that adjacent arms cannot interfere with each other. Each layer of twelve pickoff arms can patrol $100 \%$ of the field. The two motions (radius and angle) of the pickoff arms are both driven open-loop by stepper motors which are modified for cryogenic operation. LVDT encoders on each arm are used to check for successful movement. All 24 arms can be commanded to move simultaneously. A typical field re-configuration takes $\sim 4$ minutes and is accomplished whilst the telescope is slewing to the next field to avoid any additional dead time. 


\subsection{Integral Field Units and Filters}

The deployable integral field units contain a set of fore-optics that collect the output beam from each of the 24 pickoff arms and reimage it with 2:1 anamorphic magnification onto the image slicers. Immediately in front of the fore-optics is a filter wheel, which is used for order-sorting with the selected gratings. The d-IFU sub-system itself has no moving parts and uses gold-coated aluminium mirrors for optimum performance in the near-infrared and at cryogenic temperatures. The anamorphic magnification is required in order that the spatial sampling pixels (spaxels) on the sky are square whilst maintaining Nyquist sampling of the spectral line profile on the detector in the spectral dimension. All of the micro-optics in the IFUs are produced using a combination of diamond-turning and raster fly-cutting techniques (Dubbeldam et al. 2012).

\subsection{Spectrographs}

The spectrograph sub-system is comprised of three identical units, which feed three detector sub-systems (Masters et al. 2010). Each spectrograph uses a single off-axis toroidal mirror to collimate the incoming light, which is then dispersed via a reflection grating and refocused using a 6-element refractive camera. The five available gratings (IZ, YJ, $\mathrm{H}, \mathrm{K}, \mathrm{H}+\mathrm{K}$ ) are mounted on a 6 -position wheel which allows optimized gratings to be used for the individual bands. A blank position in the filter wheel is used for calibrations.

\subsection{Detectors}

KMOS uses three Teledyne substrate-removed Hawaii 2RG HgCdTe detectors with 2kx2k $18 \mu \mathrm{m}$ pixels (one detector for each spectrograph). The detectors are operated at a temperature of $35 \mathrm{~K}$ to minimize dark count and persistence (Finger et al. 2008). Typical characteristics are $\mathrm{QE}>90 \%$, readout noise $<3 \mathrm{e}^{-}$rms for long integrations with Fowler sampling, and dark current $\left.<0.003 \mathrm{e}^{-} / \mathrm{sec} / \mathrm{pixel}\right)$. Each detector is adjusted manually for tip-tilt, but is mounted on a remotely operated focus stage which was used to set the optimum focus position during commissioning. Sample-up-the-ramp (non-destructive) readout is used as standard, with Threshold Limited Integration (TLI) to extend the dynamical range for long exposure times. In this mode, if one pixel is illuminated by a bright source and reaches an absolute value above a certain threshold (close to detector saturation), only detector readouts before the threshold is reached are used to compute the slope and the counts written in the FITS image for this pixel are extrapolated to the full exposure time. This is an effective way to remove many cosmic rays on these devices.

\subsection{Infrastructure and Electronics}

The instrument housekeeping electronics (IHE) are mounted directly on the Nasmyth platform in two electronics cabinets (Bezawada et al. 2008), whilst the instrument control electronics (ICE) are mounted in three electronics cabinets which co-rotate with the instrument on the CACOR (Hess et al. 2010).

\subsection{Software}

In addition to the above hardware components, a customised data reduction pipeline has been provided for KMOS (Davies et al. 2013) which allows the observer to evaluate the data quality after each readout using real-time reconstruction of the data cubes and apply sophisticated algorithms for co-adding of data cubes and subtraction of the sky background. With over 4000 spectra per readout, automatic data processing and reduction methods have proved essential to fully exploit the scientific potential of KMOS. KMOS also has an optimised pickoff arm allocation tool, known as KARMA (Wegner et al. 2010), which links directly to the ESO observation preparation software (P2PP). 
KARMA assigns arms to targets in a prioritised way, whilst ensuring that no invalid arm positions are selected and allows the astronomer to manually reconfigure the list of allocated targets if required.

\section{Integration and Commissioning}

Following a successful laboratory test campaign (Sharples et al. 2012), and a test readiness review by ESO, KMOS was shipped to Paranal in Aug 2012 to begin reintegration and installation at VLT UT1. The main cryostat and associated electronics were transferred via air freight, whilst the larger CACOR consignment was shipped as sea cargo. KMOS was fully re-assembled and re-calibrated in the new instrument integration hall at Paranal over an 8-week period in Sep-Nov 2012 by a dedicated team of technical experts from the consortium partners, working closely with ESO personnel. After an extensive set of verification tests, the instrument was taken up the final stretch of the mountain road at walking pace, before being installed on the Nasmyth platform of UT1 (Antu). Because of the size of the CACOR (about four metres high), this item had to be lifted directly in through the dome aperture using an external crane.

\subsection{First Light}

First light with KMOS at the ESO VLT occurred on 21st Nov 2012 (Sharples et al. 2013). Initially this involved pointing the telescope at a relatively bright star and taking short exposures with one arm at a time placed at the centre of the field. All 24 arms revealed a star image close to the centre of the IFU field of view $(2.8 \times 2.8$ arcsec $)$ much to the relief of the commissioning team. Even this relatively simple observation required a large number of systems to be working together such as the real-time display which showed the positions of the target objects in the pipeline reconstructed data cubes. The real-time display is a key feature of KMOS which allows the telescope pointing to be refined by placing a subset of the pickoff arms onto bright targets which are then centered automatically using a shift and rotation of the telescope field of view (in much the same way that bright reference stars are used to align the slit masks in a multi-slit spectrograph). Once the field is aligned, these acquisition arms can then be redeployed to fainter science targets if desired.

\subsection{Astrometric Performance}

One of the first calibrations to be addressed was the astrometric positioning of the arms. This included both the mapping of the plate scale in the corrected focal plane(s) and also the local corrections (lookup tables) which are used to take out the individual variations in the $r-\theta$ drives for each arm. The latter have to be defined over the whole pie-shaped region accessible to each arm, and were based on an astrometric calibration procedure using densely populated star fields in open or globular star clusters. The absolute positioning accuracy of all the arms over the whole 7.2 diameter field was such that $83 \%$ of the targets lay within 1 spaxel $(0.2$ arcsec $)$ of the centre of the d-IFU. The repeatability was $<0.1$ arcsec.

\subsection{KMOS Throughput}

A second key performance parameter is the system throughput. KMOS has a large number of optical surfaces in the optical train (18 refractive and 15 reflective surfaces, excluding the grating and telescope mirrors) in order to deliver the performance capability of a multi-object integral field spectrograph. The throughput was measured on a number of nights using standard stars and is summarized in Fig. 2. The horizontal red (dashed) lines 

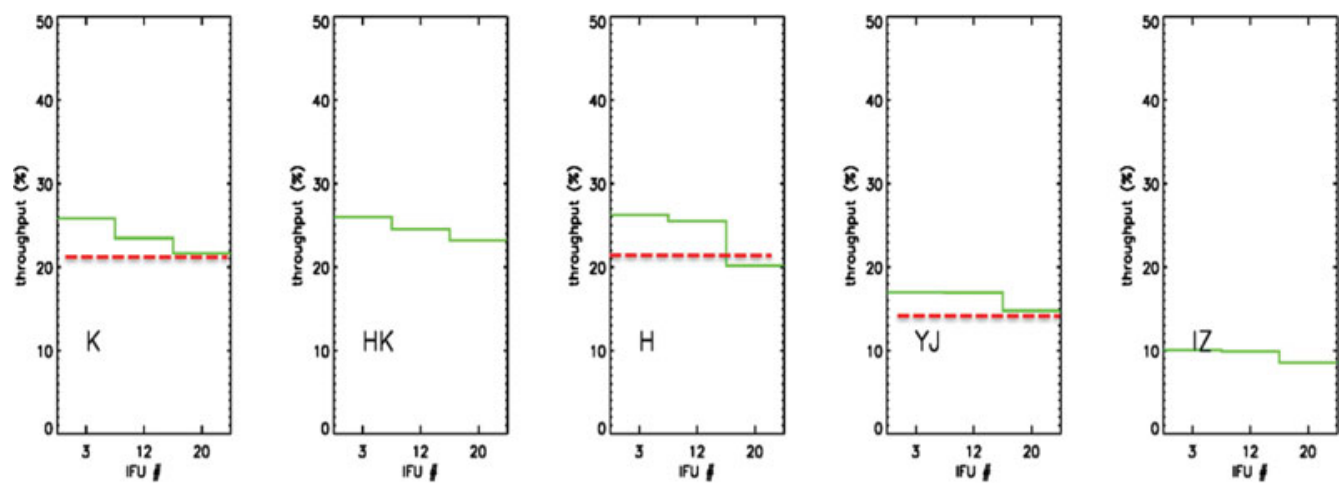

Figure 2. Total system throughput (excluding telescope and detector) in the different KMOS bands for the three different spectrographs (Spectrograph\#1 is d-IFUs 1-8, Spectrograph\#2 is d-IFUs 9-16, Spectrograph\#3 is d-IFUs 17-24)

show the minimum requirements from the instrument technical specification (Sharples et al. 2004). Since the IZ-band and HK-bands were added later, on a best-efforts basis (with no impact on the design), there are no specific requirements for these modes.

\subsection{Sky Subtraction}

One of the key performance metrics of any multi-object integral field spectrograph is the ability to do accurate sky-subtraction, particularly in the infrared bands longer than 1 micron where the night sky spectrum is dominated by extremely bright and variable $\mathrm{OH}$ emission lines. With KMOS we have experimented with various sky-subtraction strategies including conventional offsets to blank sky with a fixed d-IFU pattern, and also using dedicated sky IFUs to monitor the time-variability. Tests using long exposures of faint emission-line galaxies indicate that residuals can be reduced to $<1 \%$ except at the centres of the strongest lines, using a combination of these methods.

\section{KMOS Science}

Following three on-sky commissioning runs in Nov 2012, Jan 2013 and Mar 2013, KMOS was used for a set of science demonstration programmes proposed by the ESO community in Jun and Sep 2013. It was offered for community open time, and consortium guaranteed time, access from Oct 2013. Since then the instrument has been in heavy demand, leading to UT1 being the most oversubscribed (x6.7) telescope at Paranal in Period 92 (Oct 2013 - Mar 2014).

KMOS has now been used for a wide of scientific programmes ranging from exoplanet transit measurements through to searches for Lyman- $\alpha$ emission in extremely high redshift $(z>10)$ galaxies. As an example we show in Fig. 3 the velocity fields measured for a sample of $\mathrm{z} \sim 1$ galaxies. Previous observations of such objects have relied on single-IFU observations, making the acquisition of representative complete samples prohibitively expensive in telescope time. Observations with KMOS have already more than doubled the number of such systems with good measurements of star formation rates and internal kinematics. Also in this figure we show a H-band mosaic of Jupiter obtained by stacking 16 dithered KMOS pointings with the arms in a fixed sparse grid pattern. This produces a unique wide-field infrared integral field capability with $\sim 75,000$ spectra covering a field of $65 \times 43$ arcsec at 0.2 arcsec spatial sampling. 

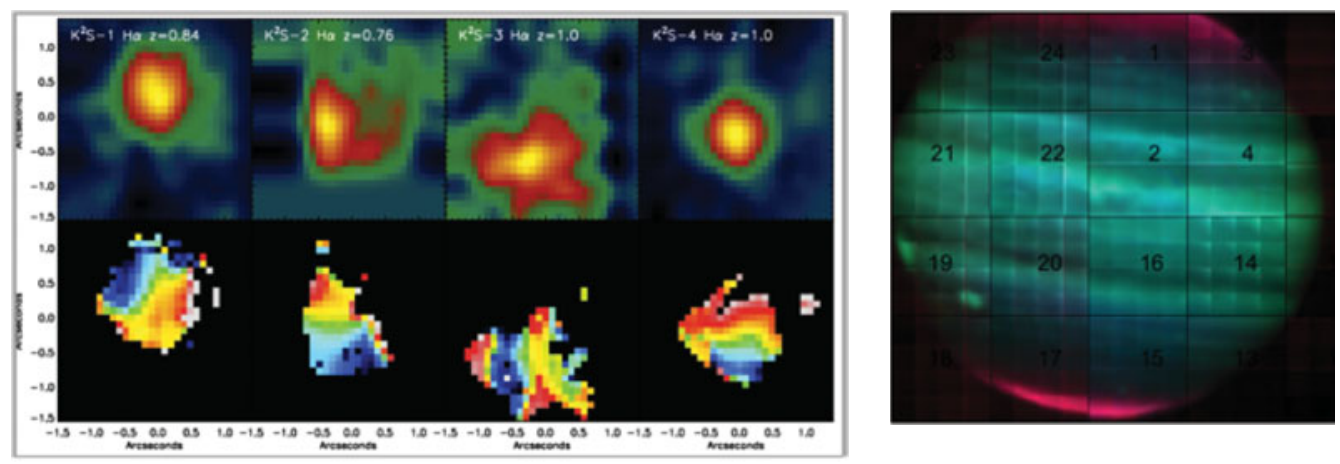

Figure 3. (left) $\mathrm{H} \alpha$ emission-line maps (upper) and derived velocity fields (lower) for a sample of faint $\mathrm{z} \sim 1$ emission-line galaxies in the GOOD-S field. The brightest targets have an observed integrated $\mathrm{H}$-alpha flux of $1.0 \times 10^{-16} \mathrm{ergscm}^{-2} \mathrm{~s}^{-1}$. These data were obtained with only $30 \mathrm{mins}$ of on-source exposure time during KMOS commissioning and demonstrate the power of this facility instrument for such surveys. (right) Reconstructed spectral image of Jupiter (pseudo colours refer to narrow bands extracted from the spectrum to highlight different molecular bands). This was created using the 24-arm mapping template with non-sidereal tracking and comprises nearly 75,000 spectra. The numbers refer to zones covered by specific d-IFUs during the dithered pointings

\section{Conclusions}

KMOS is a powerful new multi-object near- infrared integral field spectrograph now available at the ESO VLT. Its performance has been extensively verified through a series of technical and scientific tests at Paranal Observatory.KMOS is already beginning to make its mark in 3D spectroscopic surveys covering a wide variety of different science areas.

\section{Acknowledgements}

I acknowledge support from STFC grants PP/E000207/1, ST/J003034/1 and ST/K001175/1.

\section{References}

Sharples, R., et al., Proc. SPIE 5492, 1179 (2004)

Sharples, R., et al., Proc. SPIE 6269, 44 (2006)

Rees, P., et al., Proc. SPIE 7735, 166 (2010)

Dubbeldam, M., et al., Proc. SPIE 8450, 84501M (2012)

Masters, R., et al., Proc. SPIE 7735, 168 (2010)

Finger, G., et al., Proc. SPIE 7021, 20 (2008)

Bezawada, N., et al., Proc. SPIE 7014, 121 (2008)

Hess, A., et al., Proc. SPIE 7735, 93 (2010)

Davies, R., et al., Astronomy \& Astrophysics, Vol 558, A56 (2013)

Wegner, M., et al., Proc. SPIE 7740, 28 (2010)

Sharples, R., et al., Proc. SPIE 8446, 84460K (2012)

Sharples, R., et al., The Messenger 151, 21 (2013)

Davies R., MNRAS375, 1009 (2007) 